\title{
Native and Aspirin-Triggered Lipoxins Control Innate Immunity by Inducing Proteasomal Degradation of TRAF6
}

\section{Citation}

Machado, Fabiana S., Lísia Esper, Alexandra Dias, Rajat Madan, YuanYuan Gu, David Hildeman, Charles N. Serhan, Christopher L. Karp, and Júlio Aliberti. 2008. Native and aspirin-triggered lipoxins control innate immunity by inducing proteasomal degradation of TRAF6. The Journal of Experimental Medicine 205(5): 1077-1086.

\section{Published Version}

doi://10.1084/jem.20072416

\section{Permanent link}

http://nrs.harvard.edu/urn-3:HUL.InstRepos:10140326

\section{Terms of Use}

This article was downloaded from Harvard University's DASH repository, and is made available under the terms and conditions applicable to Other Posted Material, as set forth at http:// nrs.harvard.edu/urn-3:HUL.InstRepos:dash.current.terms-of-use\#LAA

\section{Share Your Story}

The Harvard community has made this article openly available.

Please share how this access benefits you. Submit a story.

Accessibility 


\title{
Native and aspirin-triggered lipoxins control innate immunity by inducing proteasomal degradation of TRAF6
}

\author{
Fabiana S. Machado, ${ }^{1}$ Lísia Esper, ${ }^{1}$ Alexandra Dias, ${ }^{1}$ Rajat Madan, ${ }^{1}$ \\ YuanYuan Gu, ${ }^{1}$ David Hildeman, ${ }^{2}$ Charles N. Serhan, ${ }^{4}$ \\ Christopher L. Karp, ${ }^{1}$ and Júlio Aliberti ${ }^{1,3}$
}

'Divisions of Molecular Immunology, ${ }^{2}$ Immunobiology, and ${ }^{3}$ Pulmonary Medicine, Cincinnati Children's Hospital Medical Center and the University of Cincinnati College of Medicine, Cincinnati, OH 45229

${ }^{4}$ Center for Experimental Therapeutics and Reperfusion Injury, Department of Anesthesia, Perioperative and Pain Medicine, Brigham and Women's Hospital and Harvard Medical School, Boston, MA 02115

Innate immune signaling is critical for the development of protective immunity. Such signaling is, perforce, tightly controlled. Lipoxins (LXs) are eicosanoid mediators that play key counterregulatory roles during infection. The molecular mechanisms underlying LXmediated control of innate immune signaling are of interest. In this study, we show that LX and aspirin (ASA)-triggered LX (ATL) inhibit innate immune signaling by inducing suppressor of cytokine signaling (SOCS) 2-dependent ubiquitinylation and proteasome-mediated degradation of TNF receptor-associated factor (TRAF) 2 and TRAF6, which are adaptor molecules that couple TNF and interleukin-1 receptor/Toll-like receptor family members to intracellular signaling events. LX-mediated degradation of TRAF6 inhibits proinflammatory cytokine production by dendritic cells. This restraint of innate immune signaling can be ablated by inhibition of proteasome function. In vivo, this leads to dysregulated immune responses, accompanied by increased mortality during infection. Proteasomal degradation of TRAF6 is a central mechanism underlying LX-driven immune counterregulation, and a hitherto unappreciated mechanism of action of ASA. These findings suggest a new molecular target for drug development for diseases marked by dysregulated inflammatory responses.

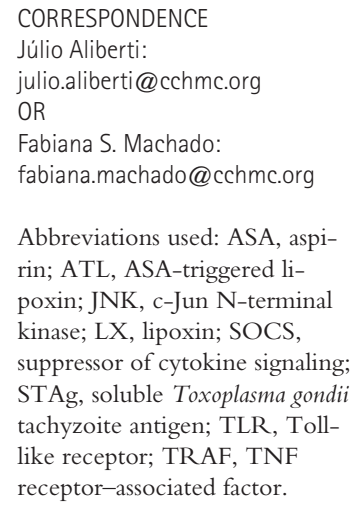

Abbreviations used: ASA, aspirin; ATL, ASA-triggered lipoxin; JNK, c-Jun N-terminal kinase; LX, lipoxin; SOCS, suppressor of cytokine signaling; STAg, soluble Toxoplasma gondii tachyzoite antigen; TLR, Tolllike receptor; TRAF, TNF receptor-associated factor.

Lipoxins (LXs) are trihydroxytetraene-containing arachidonic acid mediators that down-modulate and promote the resolution of inflammatory processes (1-7). LXs play a key counterregulatory role in mouse models of infection with Mycobacterium tuberculosis and Toxoplasma gondii $(1-3,7)$. Similarly, deficient LX-mediated counterregulation has been linked to the pathogenesis of inflammatory diseases, such as severe asthma (8), cystic fibrosis lung disease (3), and periodontal disease (7). The beneficial effects of LX analogue administration in diverse rodent models of inflammatory pathology, along with the observation that administration of the widely used antiinflammatory drug ASA leads to the generation of metabolically more stable LX carbon 15-epimers (ASA-triggered LX [ATL]), has suggested therapeutic promise for specific harnessing of the biological activities of these po-

The online version of this article contains supplemental material. tent lipid mediators. However, their molecular mechanisms of action remain underdefined.

We recently showed that suppressor of cytokine signaling (SOCS) 2 is a crucial intracellular mediator of the antiinflammatory actions of LX and ATL in vivo during T. gondii infection (9). SOCS proteins are known to inhibit receptor-mediated signal transduction via several pathways, including inhibition of tyrosine phosphorylation by allosteric blockage (e.g., of JAK proteins) and induction of proteasomal degradation through promoting polyubiquitylation (e.g., of STAT proteins) (10). Our studies were aimed at defining the molecular targets and mechanisms of action of LX- and ATL-induced SOCS2. The results presented in this study demonstrate that LX and ATL induce SOCS2-dependent ubiquitinylation and proteasomal degradation of TNF receptor-associated factor (TRAF) 2 and TRAF6, inhibiting $\mathrm{DC}$ production of proinflammatory cytokines.

() 2008 Machado et al. 
Further, these results indicate that the proteosome-dependent pathway of modulating innate immune signaling affected by LX and ATL is critical for restraining in vivo inflammatory responses during infection with $T$. gondii. Our findings elucidate a previously unappreciated mechanism of action of ASA, and suggest a new molecular target for drug development for diseases caused or exacerbated by dysregulated inflammatory responses.
RESULTS

LX-induced SOCS2 physically interacts with TRAF2 and TRAF6

Given that LX-induced SOCS2 is a physiological modulator of Toll-like receptor (TLR)-dependent innate immune recognition of $T$. gondii, we examined whether LX-induced SOCS2 interacted specifically with conserved signaling elements

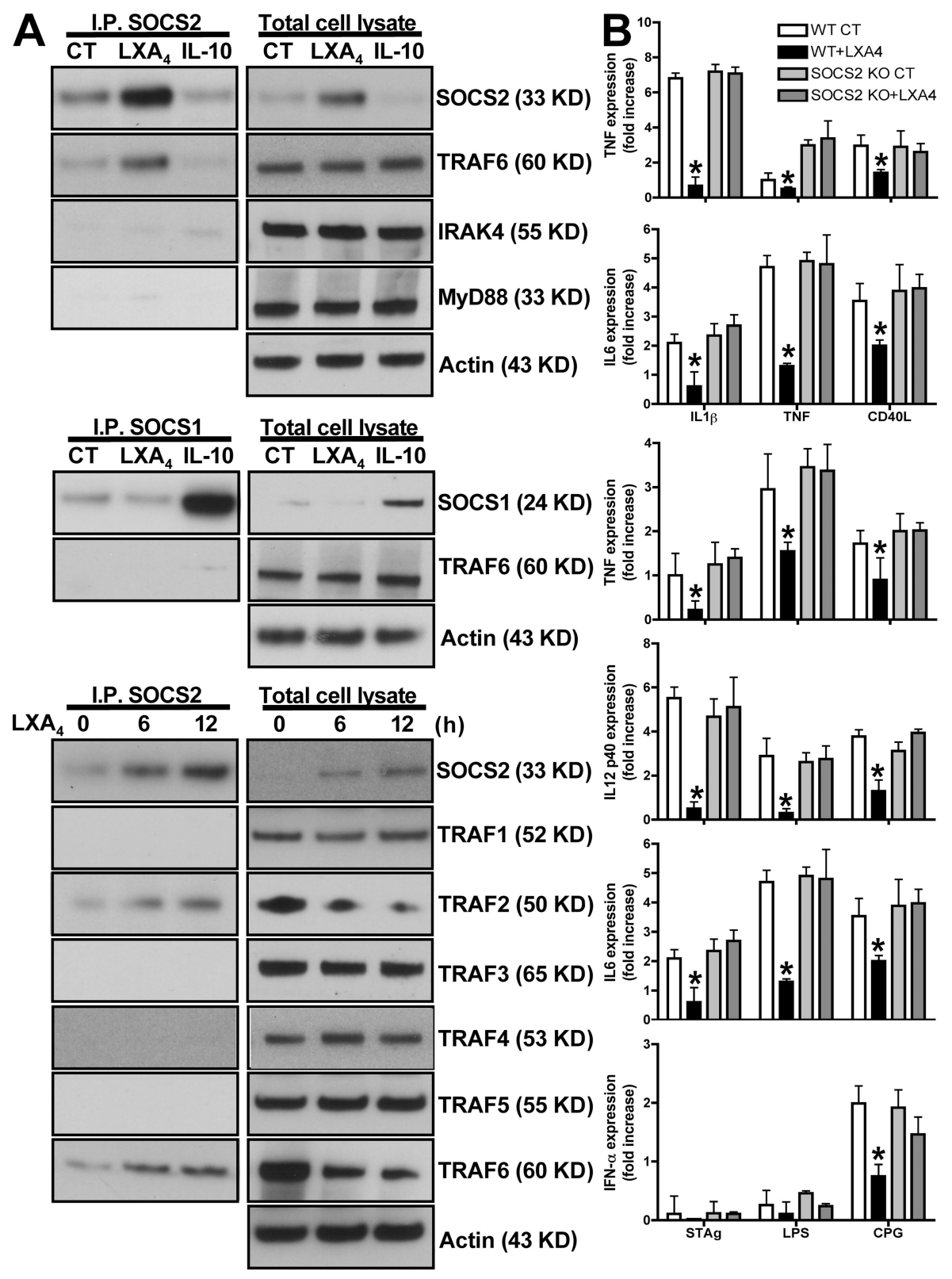

Figure 1. LX-induced SOCS2 physically interacts with TRAF2 and TRAF6, inhibiting proinflammatory cytokine expression by DCs. (A) Splenic DCs were incubated with $1 \mu \mathrm{g} / \mathrm{ml} \mathrm{LXA}_{4}$ or $100 \mathrm{ng} / \mathrm{ml} \mathrm{IL-10}$ or media control (CT) for 6 or $12 \mathrm{~h}$. SOCS2 or SOCS1 was immunoprecipitated (I.P.) from whole-cell lysates, followed by Western blot analysis of immunoprecipitates and total cell lysates to quantify SOCS1, SOCS2, TRAF1-6, IRAK4, MyD88, and actin expression. (B) DCs from WT or SOCS2-deficient mice were exposed to $1 \mu \mathrm{g} / \mathrm{ml} \mathrm{LXA}$ or vehicle for $6 \mathrm{~h}$, followed by stimulation with IL-1 $\beta$, TNF (both at $100 \mathrm{ng} / \mathrm{ml}$ ), CD40L, STAg, LPS, or CpG-oligonucleotides (all at $1 \mu \mathrm{g} / \mathrm{ml}$ ). $4 \mathrm{~h}$ later, mRNA was purified and real-time RT-PCR was used to quantify TNF, IL-6, IL-12p40, and IFN- $\alpha$ mRNA expression. Data shown are the mean ( \pm the SD) of triplicate samples, and are representative of at least two independent experiments with similar results. Asterisks indicate statistically significant differences between SOCS2-deficient and WT control mice $(P<0.05)$. 
downstream of the TLRs. Purified splenic DCs ( 90\% CD11 $c^{+}$) were incubated with $\mathrm{LXA}_{4}$ (or IL-10, which activates SOCS1 and SOCS3, as a control), and coimmunoprecipitation techniques were used to define specific interactions of TLR signaling intermediates with SOCS1 and SOCS2. As shown in Fig. $1 \mathrm{~A}$, immunoprecipitation of SOCS2, but not SOCS1, from $\mathrm{LXA}_{4}$-treated DCs led to specific coprecipitation of TRAF6 and TRAF2, but not MyD88, IRAK4, or any of the other TRAFs. This specificity among TRAFs is notable. SOCS interacts with $\mathrm{SH} 2$ domains on other proteins (10), which are present on all members of the TRAF family.
SOCS2 targeting of TRAF2 and TRAF6 inhibits proinflammatory cytokine production by DCs

TRAFs serve as critical adaptor molecules in signaling cascades downstream of receptors in the TNF and IL-1 receptor families of receptors, as well as TLRs (11-13). TRAF2 and TRAF6 regulate the transcription of downstream target genes via activation of the c-Jun N-terminal kinase (JNK) and NF$\kappa \mathrm{B}$ signaling pathways (14-19). Notably, TRAF6-deficient mice fail to produce IL-12 in response to microbial stimuli (19). To examine the functional consequences of $\mathrm{LXA}_{4^{-}}$ stimulated SOCS2-TRAF interactions during DC activation, WT and SOCS2-deficient mouse splenic DCs were

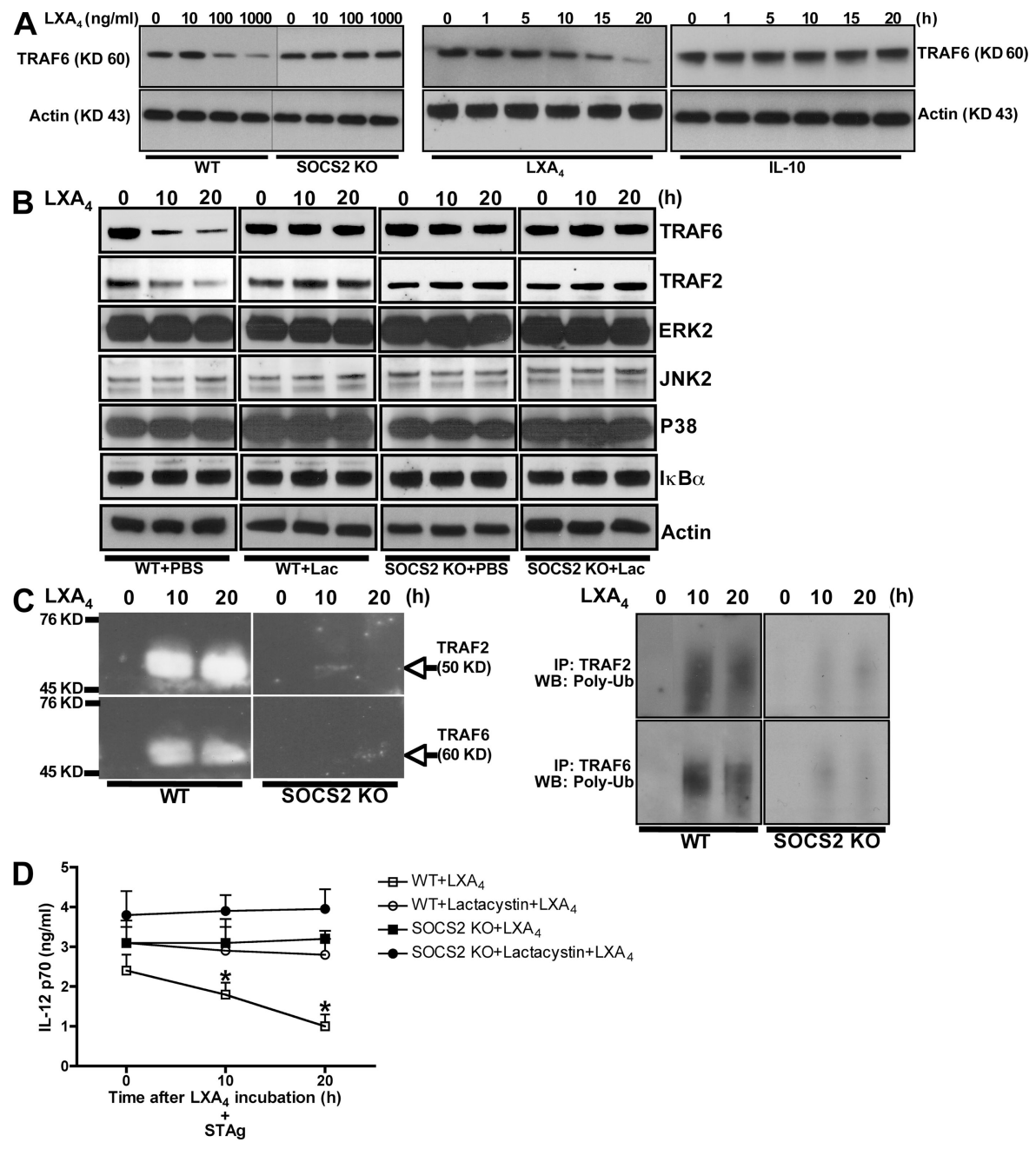

Figure 2. SOCS2 targets TRAF2 and TRAF6 for proteasomal degradation. (A) WT and SOCS2-deficient splenic DCs were treated with LXA 4 (at concentrations ranging from 10 to $1,000 \mathrm{ng} / \mathrm{ml}$ ) for $20 \mathrm{~h}$, or were treated with $\mathrm{LXA}_{4}$ or IL-10 (at $100 \mathrm{ng} / \mathrm{ml}$ times ranging from 1 to $20 \mathrm{~h}$ ), followed by quantification of TRAF6 and actin expression by Western blot. (B) DCs from WT and SOCS2-deficient mice were treated with $2 \mu \mathrm{M}$ lactacystin or medium alone, $1 \mathrm{~h}$ before incubation with $1 \mu \mathrm{g} / \mathrm{ml} \mathrm{LXA}$. At the indicated times, whole-cell extracts were prepared and TRAF2, TRAF6, ERK2, p38, JNK2, I $\mathrm{kB} \alpha$, and actin expression was quantified by immunoblot analysis. (C) DCs from WT and SOCS2-deficient mice were incubated with $1 \mu \mathrm{g} / \mathrm{ml}$ LXA . At the indicated times, whole-cell extracts were immunoprecipitated with antibodies to ubiquitin, TRAF2, or TRAF6, followed by Western blot analysis using antibodies to TRAF2, TRAF6, or polyubiquitin (clone FK1). (D) WT and SOCS2-deficient DCs were treated with $2 \mu \mathrm{M}$ lactacystin or vehicle for $1 \mathrm{~h}$. DCs were subsequently incubated with $1 \mu \mathrm{g} / \mathrm{ml} \mathrm{LXA}{ }_{4}$ or vehicle for $8 \mathrm{~h}$, followed by stimulation with $100 \mathrm{ng} / \mathrm{ml} \mathrm{STAg}$ for $24 \mathrm{~h}$. IL-12 p70 levels were quantified by ELISA in culture supernatants. Data shown represent the means \pm the SD of triplicate samples and are representative of at least two independent experiments with similar results. ${ }^{*}, \mathrm{P}<0.01, \mathrm{PBS}$ versus inhibitor treatment. 
treated with $\mathrm{LXA}_{4}$ and stimulated with IL-1 $\beta$, TNF, CD40L, LPS, CpG-oligonucleotides, or soluble T. gondii tachyzoite antigen (STAg). Exposure to $\mathrm{LXA}_{4}$ strongly blocked the expression of IL-6, TNF- $\alpha$, IL-12, and IFN- $\alpha$ in WT but not SOCS2-deficient DCs, regardless of the stimulus (Fig. 1 B). Thus, LX-induced SOCS2 inhibits biological responses known to be dependent on TRAF2/6, suggesting that these adaptors are functional targets of SOCS2-dependent modulation of proinflammatory responses.

\section{SOCS2 targets TRAF2 and TRAF6}

\section{for proteasomal degradation}

SOCS proteins mediate their actions by docking to the intracellular domains of pattern recognition, cytokine, or hormone receptors (thereby preventing interactions with downstream signaling elements), or by ubiquitinylation and proteasomedependent degradation of signaling intermediates (10, 20-22). To analyze the latter possibility, we quantified the stability of TRAF2/6 in $\mathrm{LXA}_{4}$-exposed (or IL-10-exposed) DCs. As shown in Fig. 2 (A and B), exposure to $\mathrm{LXA}_{4}$, but not IL-10, led to a SOCS2-dependent reduction in the amount of TRAF2 and TRAF6 protein expression as early as $5 \mathrm{~h}$ after exposure, whereas the expression levels of related signaling intermediates, including ERK2, JNK2, p38, and IкB $\alpha$ were not altered. Notably, $\mathrm{LXA}_{4}$ exposure led to SOCS2dependent polyubiquitylation of TRAF2 and TRAF6 (Fig. $2 \mathrm{C})$. Also, it is important to note that the reciprocal immune precipitation of TRAF2/6, followed by Western blot for polyubiquitin chain, revealed a similar pattern of LXinduced SOCS2-dependent polyubiquitylation of TRAF2 and
TRAF6 in DCs (Fig. 2 C). Thus, as might be expected, biochemical inhibition of proteasome activity with lactacystin led to parallel ablation of both LX-induced, SOCS2-dependent TRAF2/6 degradation and LX-induced, SOCS2dependent inhibition of TLR-driven cytokine production in DCs (Fig. 2, B and D). The fact that treatment of SOCS2deficient DCs with lactacystin failed to alter TRAF levels and IL-12 production (Fig. 2, B and D) suggests little toxicity for this inhibitor under these conditions. Collectively, these data suggest that these LX-mediated antiinflammatory actions are dependent on SOCS2-induced TRAF2 and TRAF6 proteasomal degradation.

\section{Restoration of TRAF6 expression overcomes LX-mediated modulation of myeloid cell cytokine production}

To directly define the roles of TRAF2 and TRAF6 as downstream targets for LX-mediated antiinflammatory activity, we analyzed the ability of $\mathrm{LXA}_{4}$ to inhibit IL-12 and TNF production in response to TLR or TNFR stimulation after overexpression of TRAF2, TRAF3, or TRAF6 in a mouse macrophage cell line (RAW264.7). As shown in Fig. 3 A, TRAF2 and TRAF6 overexpression overcame LX-dependent degradation. Only overexpression of TRAF6, but not TRAF2 or 3, reversed LX-induced inhibition of IL-12 and TNF production after TNFR/TLR stimulation (Fig. 3 B; Table I; Fig. S1, A [Table I] and B [Table II and III], available at http://www.jem.org/cgi/content/full/jem.20072416/DC1). Thus, SOCS2-mediated degradation of TRAF6 is essential to LX-mediated inhibition of proinflammatory cytokine production.
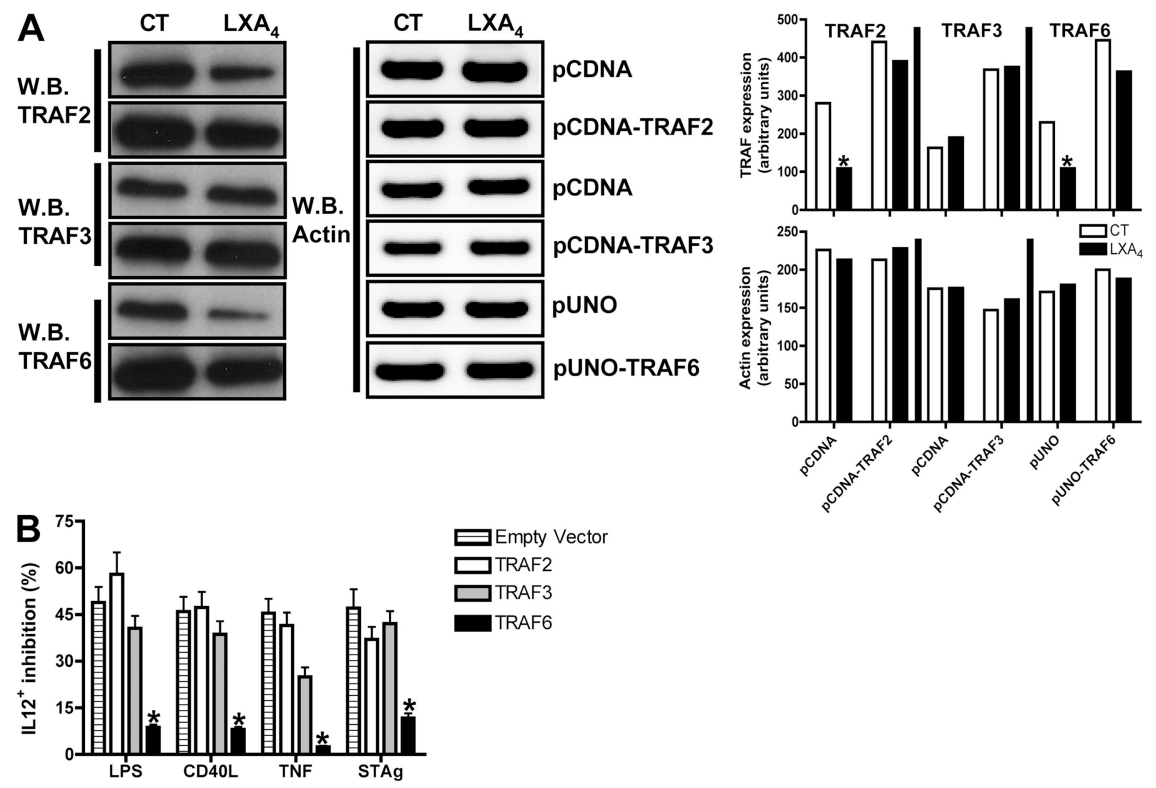

Figure 3. Restoration of TRAF expression overcomes LX-mediated modulation of myeloid cell cytokine production. (A) RAW 264.7 cells were transfected with TRAF2, TRAF3, TRAF6, or control plasmids, followed by quantification of TRAF2, TRAF3, TRAF6, and actin by Western blot. RAW 264.7 cells, transfected and treated with $\mathrm{LXA}_{4}$ as noted in B, were stimulated with TNF (100 ng/ml), CD40L, STAg or LPS (all at $\left.1 \mu \mathrm{g} / \mathrm{ml}\right)$. $24 \mathrm{~h}$ later, IL-12p70 was quantified by FACS. Data shown represent the means \pm the SD of triplicate samples and are representative of at least two independent experiments with similar results. ${ }^{*}, \mathrm{P}<0.01$, PBS versus $\mathrm{LX}$ treatment. 
Table I. Restoration of TRAF expression overcomes LX-mediated modulation of myeloid cell cytokine production

\begin{tabular}{lccccccccc}
\hline GFP+IL12+ (\%) & PBS & LPS & LPS + LXA & CD40L & CD40L $+L X A$ & TNF & TNF+LXA & STAg & STAg $+L X A$ \\
\hline Empty Vector & $1.5 \pm 0.18$ & $7.48 \pm 0.9$ & $3.82 \pm 0.41$ & $8 \pm 0.87$ & $4.32 \pm 0.36$ & $9.12 \pm 0.97$ & $4.47 \pm 0.58$ & $11 \pm 1.4$ & $5.82 \pm 0.9$ \\
TRAF2 & $2 \pm 0.31$ & $8.88 \pm 1$ & $3.73 \pm 0.47$ & $6.37 \pm 0.59$ & $3.36 \pm 0.49$ & $9.4 \pm 0.89$ & $5.5 \pm 0.63$ & $12.7 \pm 1.5$ & $8 \pm 0.92$ \\
TRAF3 & $1.8 \pm 0.4$ & $15 \pm 2.1$ & $8.91 \pm 0.9$ & $10.3 \pm 1.5$ & $6.36 \pm 0.9$ & $16.5 \pm 2.9$ & $12.38 \pm 1.2$ & $12.5 \pm 2$ & $7.23 \pm 2.8$ \\
TRAF6 & $1.6 \pm 1$ & $11.4 \pm 2.2$ & $10.4 \pm 0.8$ & $10.7 \pm 1.9$ & $9.83 \pm 1.4$ & $12 \pm 2.5$ & $11.3 \pm 1.7$ & $13.8 \pm 2.1$ & $12.18 \pm 1.7$ \\
\hline
\end{tabular}

$10^{7}$ RAW 264.7 cells were transfected with mTRAF6, flag-tagged mTRAF2, flag-tagged mTRAF3, or the respective control plasmids (10 $\left.\mu \mathrm{g}\right)$, followed by incubation in 24-well plates for $15 \mathrm{~h}$. Cells were then exposed to LXA $(1 \mu \mathrm{g} / \mathrm{ml})$ or vehicle, and incubated for a further $24 \mathrm{~h}$ before stimulation with LPS, CD 40L, STAg (all at $1 \mu \mathrm{g} / \mathrm{ml})$, and TNF (at $100 \mathrm{ng} / \mathrm{ml})$. IL-12 p70 expression was subsequently quantified by FACS.

Proteasome inhibition abolishes the in vivo antiinflammatory actions of LX-stimulated SOCS2: modulation of proinflammatory responses to microbial challenge

To assess the in vivo relevance of LX-induced, SOCS2-dependent TRAF6 proteasomal degradation, we moved to an in vivo model of LX-dependent inhibition of DC IL-12 production, termed "DC paralysis" (23). In this system, injection of STAg causes endogenous generation of $\mathrm{LXA}_{4}$ that, in turn, inhibits IL-12 production by DCs during subsequent rechallenge (Fig. 4 A). As noted previously, DC paralysis is SOCS2-dependent; SOCS2-deficient mice produce equivalent amounts of IL-12 regardless of previous STAg challenges (Fig. 4 A) (9). Importantly, mice treated with lactacystin or another proteasome inhibitor, PR-11 (24), failed to exhibit DC paralysis, producing equivalent levels of IL-12 as single STAg-injected WT mice (Fig. 4 A). Moreover, neither lactacystin nor PR11 treatment affected IL-12 production in STAg-injected SOCS2-deficient mice, excluding the possibility of unrelated nonspecific effects after in vivo proteasome inhibition. IL-10-deficient mice suffer acute mortality caused by uncontrolled inflammation during T. gondii infection (25). The induction of DC paralysis rescues IL-10deficient mice from such mortality (25). Notably, pretreatment with the proteasome inhibitor PR11 before DC paralysis ablated the protective effects of STAg-induced DC paralysis in IL-10-deficient mice (Fig. 4 B). Thus, the protective actions of LX-stimulated DC paralysis during T. gondii infection are mediated in a proteasome-dependent manner that correlates with SOCS2-driven proteasome-mediated degradation of TRAF6.

\section{In vivo proteasome inhibition abolishes}

the antiinflammatory actions of LX-stimulated SOCS2: modulation of proinflammatory responses during infection Endogenously generated LXs control proinflammatory responses through SOCS2 during $T$. gondii and $M$. tuberculosis infection $(2,26)$. In the absence of LXs or SOCS2, infection with $T$. gondii is lethal because of aberrant, uncontrolled inflammation (26). To analyze whether proteasomal degradation of TRAF2 and TRAF6 are downstream targets for LX-mediated control of proinflammatory responses during in vivo infection, we infected WT and SOCS2-deficient mice with T. gondii, in the presence or absence of treatment with PR11 starting at day 11, which was previously found to be the time at which increases in serum levels of $\mathrm{LXA}_{4}$ are detectable. At this time point, most $\mathrm{T}$ cell priming is thought to have occurred. Moreover, direct testing of the effect of proteasome inhibition on $\mathrm{CD}^{+} \mathrm{T}$ cell during LCMV infection showed that such treatment did not affect expansion of $\mathrm{CD}^{+} \mathrm{T}$ cells specific for LCMV-derived peptides (Fig. S2, available at http://www.jem.org/cgi/content/full/jem.20072416/DC1). As shown in Fig. $5 \mathrm{~A}$, proteasome inhibition during $T$. gondii infection mirrored the uncontrolled proinflammatory phenotype seen in both 5-LO- and SOCS2-deficient mouse strains $(9,26)$, including: (a) high levels of systemic (Fig. 5, A and B) and tissue (Figs. S3-S5) proinflammatory cytokine production; (b) more efficient control of parasite burden (Fig. 5 E); and

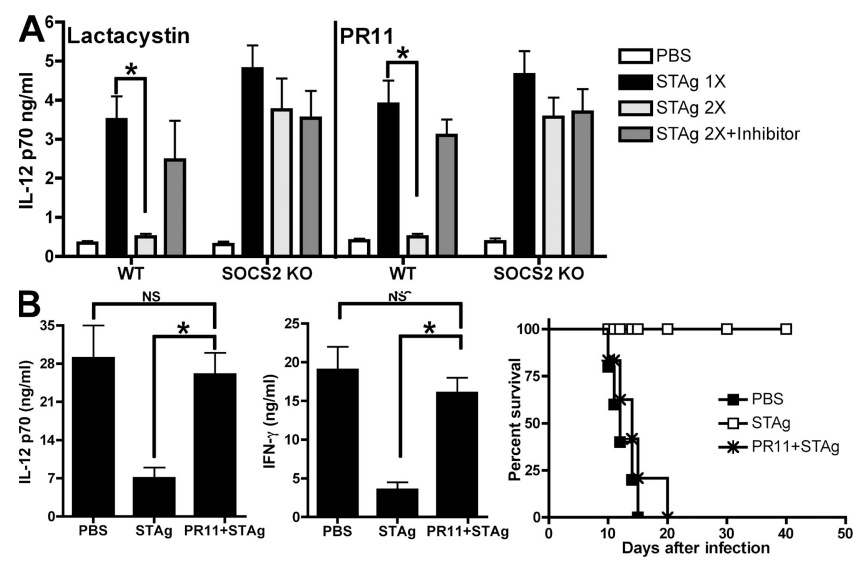

Figure 4. In vivo proteasome inhibition abolishes the antiinflammatory actions of LX-induced SOCS2; inhibition of microbial product challenge. (A) WT and SOCS2-deficient mice ( $n=5$ mice/group) were injected intraperitoneally with $0.2 \mathrm{ml} /$ mouse PBS, $20 \mathrm{mg} / \mathrm{kg}$ lactacystin, or $0.2 \mathrm{mg} / \mathrm{kg}$ PR $11.1 \mathrm{~h}$ later, animals were challenged with PBS or STAg ( $5 \mu \mathrm{g} /$ mouse). $24 \mathrm{~h}$ later, mice were rechallenged with STAg. $6 \mathrm{~h}$ later, splenic DCs were harvested, purified, and cultured overnight to allow for spontaneous cytokine release. IL-12p70 secretion was quantified by ELISA. (B) IL10-deficient mice ( $n=5$ mice/group) were injected intraperitoneally with PBS $(0.2 \mathrm{ml} / \mathrm{mouse})$ or PR $11(0.2 \mathrm{mg} / \mathrm{Kg})$, and after $1 \mathrm{~h}$, with PBS or STAg $(5 \mu \mathrm{g} / \mathrm{ml}) .24 \mathrm{~h}$ later, mice were infected intraperitoneally with 20 cysts of T. gondii (ME49 strain). 5 and $7 \mathrm{~d}$ after infection, serum concentrations of IL-12 p70 and IFN- $\gamma$ were quantified by ELISA; mortality was monitored over time. Graphed values represent the means \pm the SD. The experiments shown are representative of at least two independent experiments yielding similar results. 

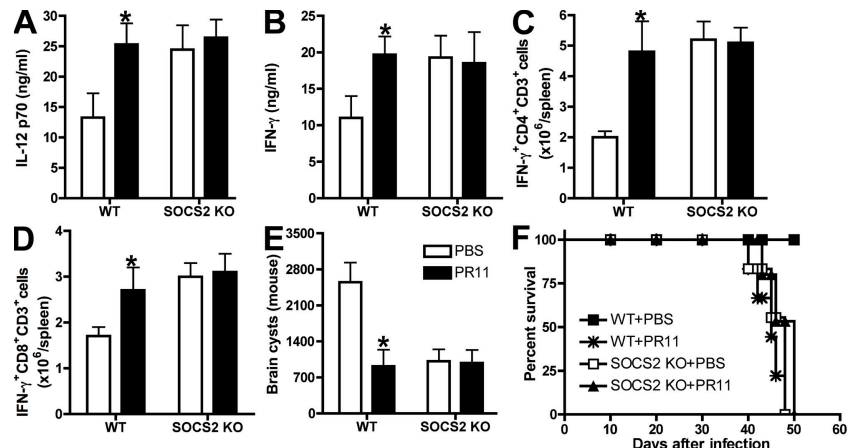

G
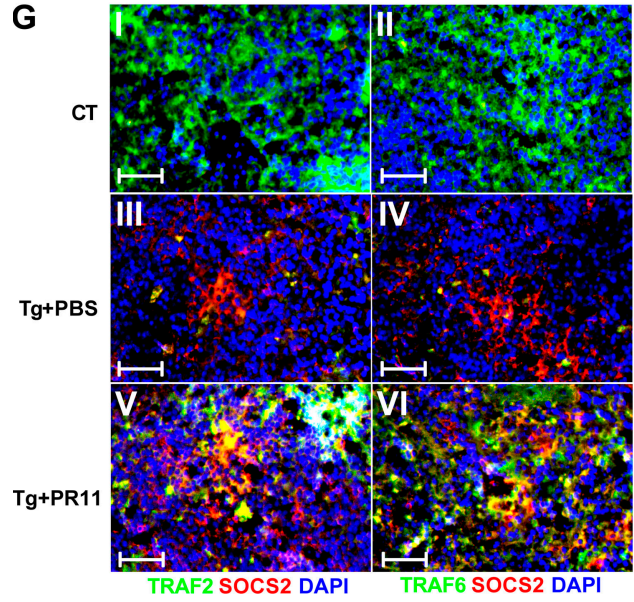

Figure 5. In vivo proteasome inhibition abolishes the antiinflammatory actions of LX-induced SOCS2: modulation of proinflammatory response during infection. (A) WT and SOCS2-deficient mice ( $n=$ 10/group) were infected intraperitoneally with $T$. gondii, and treated with PBS (0.2 ml/mouse) or PR 11 ( $0.2 \mathrm{mg} / \mathrm{kg}$ ) every other day, from 11 to $27 \mathrm{~d}$ after infection. Serum concentrations of IL-12 p70 (A) and IFN- $\gamma(B)$ were quantified by ELISA $15 \mathrm{~d}$ after infection. At the same time point, mice were killed, spleens were harvested, and cell suspensions were prepared and incubated with PBS or STAg (100 ng/ml) for $4 \mathrm{~h}$, followed by flow cytometric quantification of intracellular IFN- $\gamma$ in $\mathrm{CD}_{4}^{+}(\mathrm{C})$ and $\mathrm{CD}^{+}$ $T$ cells (D). $30 \mathrm{~d}$ after infection, the number of brain cysts was determined by microscopy (E). Mortality curves were monitored (F). (G) Spleens were removed from mice $30 \mathrm{~d}$ after infection. Frozen sections of spleens were processed and for in situ detection of SOCS2 (I-VI), TRAF2 (I, III and V), and TRAF6 (II, IV and VI) specific primary antibodies were used, followed by a secondary antibody anti-lgG Alexa Fluor 488 (green) and anti-lgG Alexa Fluor 594 (red), and the cells were counterstained with DAPI. Images were captured using an Axiovert microscope with AxioVision software (see Materials and methods). CT, uninfected control; Tg, T. gondii-infected. Graphed values represent the means \pm the SD. The experiments shown are representative of at least two independent experiments yielding similar results. Bars, $50 \mu \mathrm{m}$.

(c) mortality occurring $\sim 45-50 \mathrm{~d}$ after inoculation (Fig. $5 \mathrm{~F}$ ). Interestingly, we found higher frequencies of IFN $-\boldsymbol{\gamma}$-producing $\mathrm{CD}^{+}$and $\mathrm{CD} 8^{+} \mathrm{T}$ cells after in vitro recall stimulation (Fig. 5, C and D), supporting our findings that proteasome inhibition not only did not inhibit $\mathrm{T}$ cell priming during infection, but enhanced it. Immunofluorescence analysis revealed that SOCS2-dependent loss of TRAF2 and TRAF6 expression in spleen and brain during $T$. gondii infection was reversed by PR11 treatment (Fig. 5 G and Figs. S6-S9). It is important to emphasize that proteasomal inhibition did not affect STAg-driven proinflammatory responses in SOCS2deficient mice and DCs, suggesting that the phenotype seen in WT mice is not derived from unrelated actions by the drug. Collectively, these data clearly establish TRAF2 and TRAF6 as critical downstream targets for proteasomal degradation mediated by LX-stimulated SOCS2. Thus, LX-mediated degradation of these key proinflammatory signaling adaptor proteins is essential for tight control of the innate immune response to infection with $T$. gondii.

The antiinflammatory actions of ATLs require SOCS2dependent proteasomal degradation of TRAF2 and TRAF6 The beneficial biological effects of in vivo ASA administration occur, in part, through the generation of endogenous ATL. Our data at this juncture suggested that ATL-stimulated, SOCS2-mediated, proteasome-dependent degradation of TRAF proteins is mechanistically important for ASA-driven modulation of proinflammatory cytokine production. Indeed, similar to the effects of $\mathrm{LXA}_{4}$ exposure, in vivo exposure of DCs to ASA led to SOCS2-independent ATL generation (Fig. S10 A, available at http://www.jem.org/cgi/content/ full/jem.20072416/DC1), along with a SOCS2-dependent specific decrease in DC expression of TRAF6 and TRAF2 (Fig. 6 A). LPS treatment alone did not change baseline levels of signaling molecules either in WT or SOCS2-deficient DCs, except for the expected decrease in IкB $\alpha$ (Fig. 6 A) (24). ASA treatment before LPS exposure delayed the appearance of phosphorylated ERK1/2 and p38 in WT, but not in SOCS2-deficient, DCs (Fig. 6 A). Consistent with findings in cells exposed to ASA alone, TRAF2 and TRAF6 expression was decreased in LPS-stimulated, ASA-treated WT, but not SOCS2-deficient, DCs. Finally, LPS-induced I $\mathrm{B} \alpha$ degradation was prevented by ASA treatment in a SOCS2dependent manner (Fig. 6 A). Although the cause-effect relationship is likely to be indirect, the delay in the generation of 15-epi-LX in aspirin (ASA)-treated, COX1-deficient mice indicated that both SOCS2 induction and TRAF6 degradation are dependent on the endogenous generation of 15epi-LX (Fig. 6 B). As indicated, although both WT and SOCS2-deficient DCs generated significant levels of 15-epi-LX after ASA treatment, COX1-deficient mice presented a significant delay with low levels of the eicosanoid (Fig. S10 B). This finding suggests that COX1-derived prostanoids are required to establish the enzymatic pathways needed to produce 15-epi-LXA 4 , rather than direct involvement of COX1 in the biosynthesis of 15 -epi-LXA $\mathrm{LX}_{4}$ in this system (for review see [7]). ASA caused a SOCS2-dependent ablation of LPSinduced TNF and IL-12p70 production (Fig. 6 C), suggesting that ATL-induced SOCS2 regulates the LPS-driven signaling cascade in DCs by inhibiting I $\mathrm{KB} \alpha$ degradation and abolishing NF- $\kappa B$ signaling. In agreement with these observations, in vivo treatment with ASA led to TRAF2/TRAF6 polyubiquitylation and degradation that could be prevented by pretreatment with PR11 (Fig. 6, D and E). Such treatment 


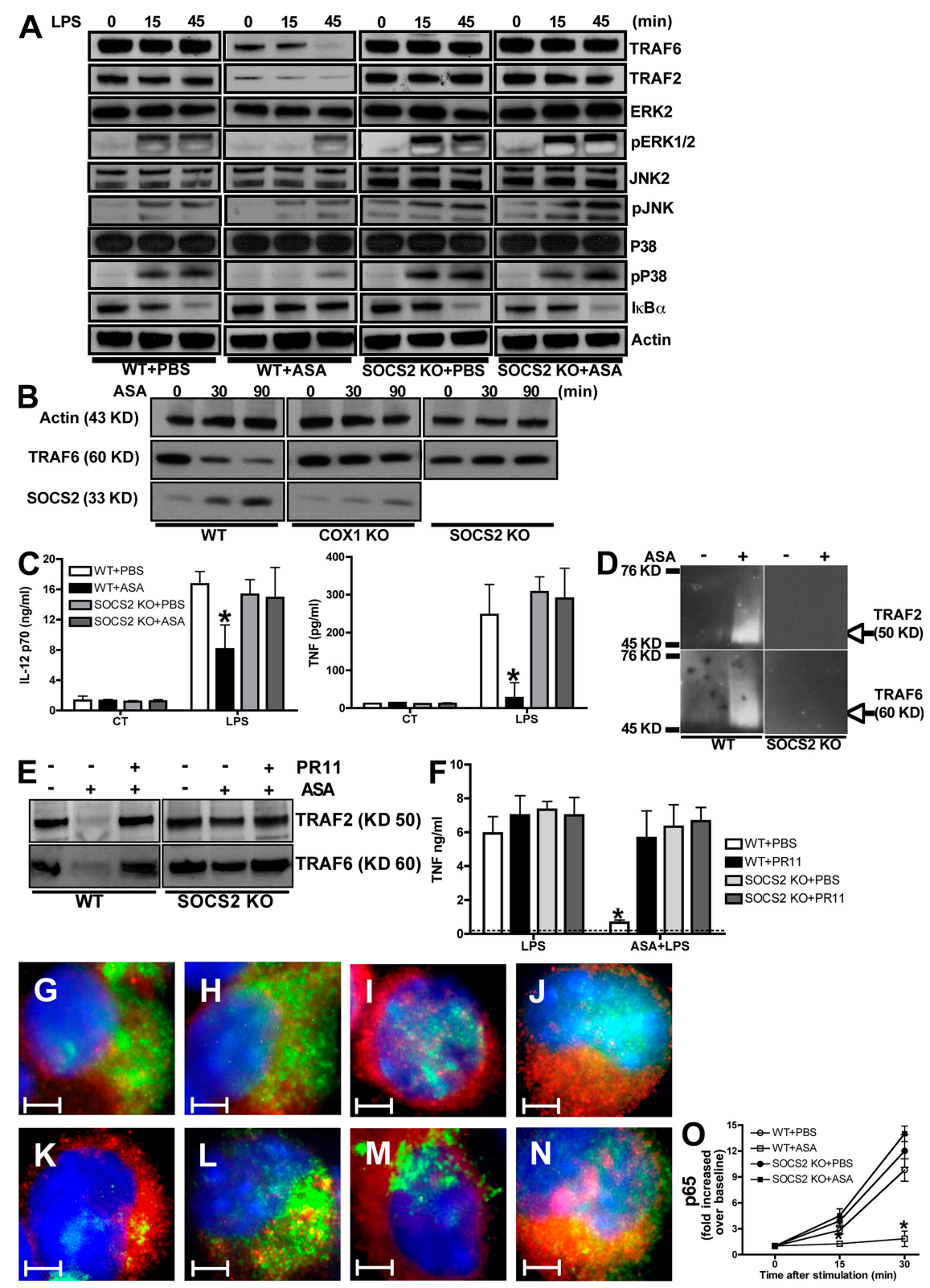

Figure 6. The antiinflammatory actions of ATLs require SOCS2-dependent proteasomal degradation of TRAF2 and TRAF6. (A) WT and SOCS2deficient mice ( $n=10$ mice/group) were treated orally with ASA ( $5 \mathrm{mg} / \mathrm{kg}$ ), or PBS as a control. 45 min later, splenic DCs were purified and stimulated with LPS $(1 \mu \mathrm{g} / \mathrm{ml})$ or medium alone. At the indicated times, TRAF2, TRAF6, ERK2, p38, JNK2, I $\kappa$ B $\alpha$, pJNK, pERK1/2, pP38, and actin expression were quantified as indicated in Fig. 2. (B) Splenic DCs purified from WT, SOCS2-deficient, and COX1-deficient mice were incubated in the absence or presence of $5 \mathrm{mM}$ ASA for 0, 30, or $90 \mathrm{~min}$, followed by quantification of actin, TRAF6, and SOCS2 expression by Western blot. (C) Splenic DCs, purified from WT and SOCS2-deficient mice that had been treated with ASA or PBS as in A, were stimulated with LPS $(1 \mu \mathrm{g} / \mathrm{ml})$ or PBS. After overnight culture, IL-12p70 and TNF secretion was quantified in by ELISA. (D) Splenic DCs purified from WT and SOCS2-deficient mice that had been treated with ASA or PBS as in A underwent Western blot analysis of polyubiquitylated TRAF2 and TRAF6, as indicated in Fig. 2. (E and F) WT and SOCS2-deficient mice were injected intraperitoneally with $0.2 \mathrm{mg} / \mathrm{Kg}$ PBS or PR11, followed by PBS or ASA treatment. $1 \mathrm{~h}$ later, splenic DCs were purified and whole-cell extracts underwent immunoblot analysis to quantify TRAF2 and TRAF6 expression (E). WT and SOCS2-deficient mice were injected intraperitoneally with PR11 (or PBS), followed by ASA (or PBS). 45 min later, mice were challenged intraperitoneally with LPS ( $5 \mu \mathrm{g} /$ mouse). $3 \mathrm{~h}$ later, serum TNF concentrations were quantified by ELISA (F). (G-O) WT (G, I, K and M) and SOCS2-deficient ( $\mathrm{H}, \mathrm{J}, \mathrm{L}$, and N) DCS, purified from PBS-treated (F-I) or ASA-treated (J-M) mice, were incubated for 45 min with PBS $(G, H, K$, and L) or LPS $(1 \mu \mathrm{g} / \mathrm{ml} ; \mathrm{l}, \mathrm{J}, \mathrm{M}$, and $\mathrm{N})$, and then cytospun onto slides. DCs subsequently underwent immunofluorescence staining for TRAF6 (green) and p65 (red), followed by counterstaining with DAPI (blue). Microphotographs were taken using an Axiovert microscope with AxioVision software (see Materials and methods). (0) Nuclear extracts were prepared and the levels of DNA-bound p65 quantified using an NF-кB p65 Transcription Factor Assay kit. Data shown are the mean \pm the SD of triplicate samples, and are representative of three independent experiments with similar results. ${ }^{*}, \mathrm{P}<0.01, \mathrm{PR} 11$ or ASA versus PBS treatment. Bars, $2 \mu \mathrm{m}$. 
also abolished SOCS2-dependent inhibition of in vivo LPSinduced TNF production (Fig. 6 F) and thioglycollate-stimulated neutrophil migration (Fig. S10, C and D).

To further evaluate ATL-induced, SOCS2-mediated inhibition of NF- $\kappa \mathrm{B}$ activation, we correlated NF- $\mathrm{NB}$ localization with DNA-binding activity. As shown in Fig. 6 (G-O), LPS treatment caused nuclear translocation of NF- $\mathrm{B}$ p 65 , something prevented by ASA pretreatment in a SOCS2dependent manner. NF-кB p65 DNA-binding activity paralleled its nuclear translocation (Fig. $6 \mathrm{O}$ ); similar findings were obtained for NF-кB p50 (Fig. S11, available at http://www .jem.org/cgi/content/full/jem.20072416/DC1). Thus, the antiinflammatory effects of ASA on DCs is mediated by SOCS2dependent proteasomal degradation of TRAF2/TRAF6.

\section{DISCUSSION}

Immunologists have traditionally focused on the mechanisms that underlie and control the activation of inflammatory responses. The last decade has seen growing awareness and experimental attention paid to the fact that restraint and resolution of inflammatory responses is also, perforce, under tight, active regulation. In turn, this has led to growing interest in facilitation of resolution (as opposed to inhibition of activation) as a therapeutic strategy for the dysregulated inflammation seen as central to the pathogenesis and expression of an increasingly wide spectrum of diseases (27). Therapeutic harnessing of the multilevel, resolution-promoting bioactivities of the LXs, and novel resolution mediators such as the D- and E-series resolvins and protectins, is thus quite attractive.

The mechanisms of action of the LXs, at the level of cellular signaling, are of interest. Along these lines, $\mathrm{LXA}_{4}$ and ATL restrain innate and acquired immune responses via stimulating SOCS2 expression in an AhR-dependent manner (9). The current studies elucidate new molecular targets and mechanism of action of LX/ATL-induced SOCS2. Specifically, these results indicate that: (a) LX-induced SOCS2 targets TRAF2 and TRAF6 for proteasomal degradation; (b) degradation of TRAF6 renders cells exposed to LX unable to respond to stimulation through TLRs, TNF-R, IL-1R, and CD40, resulting in the inhibition of proinflammatory cytokine production by DCs; (c) proteasome-mediated degradation of TRAF6 plays a major role in the mechanism of action of ASA in restraining DC production of proinflammatory cytokines; and (d) this pathway of targeted proteasomal degradation of signaling intermediates is critical for successful modulation of inflammatory responses during infection with the ubiquitous protozoan pathogen, T. gondii. The biological role of LX- and ASA-driven TRAF2 degradation remains to be defined.

A fundamental question in host-pathogen interactions is whether the immune system is properly activated to eradicate the pathogens, and whether this response is appropriately controlled to prevent damage to the host during control or elimination of infection. Microbial recognition is a critical first step. Central to both innate and adaptive immunity, DCs signal the presence of microbes via pattern recognition recep- tors such as the TLRs (28-31), responding by secreting cytokines important in immune activation and specification of the class of immune response. During infection with T. gondii (and infection with diverse other intracellular pathogens), DC production of IL-12, IFN- $\gamma$, and TNF is essential in controlling pathogen growth (32-34). IL-12 and TNF are potentially toxic when produced in excess, and their production is known to be carefully regulated by a remarkably large number of different mechanisms $(26,29,32)$. In toxoplasmosis, the proinflammatory process is counterbalanced by the simultaneous induction of critical, nonredundant counterregulatory mediators, including IL-10 and LXs $(32,33)$. Mice with a genetic deficiency in LX production succumb to lethal inflammatory responses during toxoplasmosis. In turn, the biological importance and nonredundant nature of the proteasome pathway to the mechanism of action of LXs is underscored by the finding that proteasome inhibition during toxoplasmosis prevents the regulatory actions of LX, restoring TLR and cytokine receptor signaling in vivo and causing uncontrolled proinflammatory cytokine production, aberrant leukocyte infiltration and elevated mortality.

Since its first chemical synthesis and clinical trials in the late 19th century, the cellular and molecular mechanisms of action of ASA have been the focus of intense research (35). In the early 1970 s, a series of reports by J.R. Vane established that ASA and ASA-like drugs inhibit prostaglandin synthesis (36). For the next $20 \mathrm{yr}$, this was the dominant view for the overall mechanism of action of ASA, despite the finding that at the cellular level, ASA inhibits neutrophil activation, even in the presence of cyclooxygenase products (37). Claria and Serhan (38) showed that, in the presence of ASA, cyclooxygenase- 2 is acetylated, yet remains active and converts arachidonic acid into intermediates that are converted into potent ATL, thus opening the possibility that ATLs are indeed mediating the antiinflammatory actions of ASA in vivo. Indeed, ATLs display powerful pro-resolution and antiinflammatory actions in several cell types and disease settings (27). This new body of evidence sheds light on the possible intracellular pathways that are controlled by ASA and ATLs. ASA has been shown to inhibit neutrophil and macrophage activation, calcium flux, and integrin activation, as well as IL-12, TNF, and IL-8 production (27), suggesting that the downstream inhibition targets are critical elements of a general proinflammatory pathway. In agreement with this hypothesis, it was shown that ASA blocks NF- $\mathrm{KB}$ activation and nuclear translocation (39). Our earlier observation that ATL-induced SOCS2 mediated some of the antiinflammatory actions of ASA (9) opened the possibility of exploring novel intracellular mechanisms of action of this drug. The results presented in this study clearly indicate that TRAF6 is a key molecular target of ASA and that proteasome-mediated degradation of TRAF6 plays an important role and is a major inflammation resolution pathway up-regulated by ASA. In a similar vein, Paul-Clark et al. recently demonstrated that 15-epi-LXA 4 mediates ASA induction of nitric oxide, negatively regulating leukocyte-endothelium interactions (40). What role this 
pathway plays in modulating DC function is currently under active investigation.

It has become clear in recent years that dysregulated inflammatory responses are central to the pathogenesis of a wide range of diseases, causing an enormous burden of morbidity and mortality in the world at large. The results presented in this study suggest that degradation of TRAF6 provides a promising biochemical target for drug development aimed at modulating inflammatory processes in these diseases.

\section{MATERIALS AND METHODS}

Animals. WT C57BL/6 mice were obtained from The Jackson Laboratory. C57BL/6, SOCS2-KO, and IL-10-KO were bred and maintained under pathogen-free conditions at the Cincinnati Children's Hospital Medical Center (CCHMC) animal facility. All experimental protocols were approved by the CCHMC Institutional Animal Care and Use Committee. Animals were infected by intraperitoneal injection with 20 cysts of $T$. gondii cysts (ME49 strain) recovered from brain homogenates from chronically infected mice. STAg was prepared from sonicated tachyzoites, as previously described (41).

Cytokine quantification. IL-12 p70 (BD Biosciences), TNF- $\alpha$, and IFN- $\gamma$ secretion was quantified using commercial ELISA kits (R\&D Systems). For in vitro experiments, splenic DCs were partially purified, as previously described (29). Cytokine concentrations in supernatants were normalized to foldchanges, based on the ratio of values in experimental to baseline samples. It is important to note that baseline cytokine secretion by naive DCs did not differ significantly among the different genetic backgrounds (WT vs. SOCS2 KO) used in these studies.

15-epi-LXA 4 . Mice or culture DCs were treated with ASA (in vivo or in vitro, respectively), and the 15 -epi-LXA 4 was quantified in urine or supernatants, respectively, using commercial ELISA kit (Neogen Corp.).

Microscopy. Spleens and brains were removed from mice before or $30 \mathrm{~d}$ after infection, and frozen sections were processed and stained with a goat antimouse SOCS2, goat anti-mouse TNF, goat anti-mouse $\mathrm{p} 50$ or $\mathrm{p} 65$, rabbit anti-mouse TRAF2 or TRAF6, and rabbit anti-mouse iNOS (all from Santa Cruz Biotechnologies), followed by a double incubation with Alexa Fluor 488-conjugated anti-mouse CD11c (Caltag), or Alexa Fluor 488-conjugated anti-rabbit and Alexa Fluor 594-conjugated anti-goat antibodies (Invitrogen), as previously described (23). The cells were counterstained for nuclei with DAPI (Invitrogen). For in situ TRAF2, TRAF6, p50, and p65 analysis, we isolated DCs (purity: $\sim 90 \% \mathrm{CD}_{11} \mathrm{c}^{+}$cells), stimulated them as described for each experiment, and fixed them with ice-cold acetone for $1 \mathrm{~min}$ and processed and stained as described above. Images were acquired using a microscope (Axiovert; Carl Zeiss, Inc.) with the AxioVision software (Carl Zeiss, Inc.)

Measurement of p50 and p65. Nuclear extracts were purified, and p50 and p65 binding activity was quantified using Transcription Factor Assay kits according to the manufacturer's instructions (ActiveMotif).

Cell extracts and immunoblots. Whole-cell extracts were generated by lysing WT and SOCS2-deficient DCs in buffer containing $50 \mathrm{mM}$ Hepes, $\mathrm{pH} 7.9,0.25 \mathrm{M} \mathrm{NaCl}, 5 \mathrm{mM}$ EDTA, 0.1\% NP-40, $1 \mathrm{mM}$ PMSF, and $1 \mathrm{X}$ HALT protease inhibitor cocktail (Thermo Fisher Scientific). For detection of phosphorylated-proteins, $\mathrm{Na}_{3} \mathrm{VO}_{4}$ and $\mathrm{NaF}$ were added to lysis buffers. Immunoprecipitation was performed with SOCS2 antibody obtained from Santa Cruz Biotechnology, Inc. The detection of protein expression by Western blot was performed with primary antibodies against SOCS2, SOCS1, TRAF1-6, IRAK4, MyD88, ERK2, P38, JNK2, IкB $\alpha$, pJNK, and pERK1/2, all obtained from Santa Cruz Biotechnology Inc. Anti-phospho-SAPK/JNK and anti-phospho-p38 antibodies were purchased from Cell Signaling Technology, and anti-JNK/SAPK1 was purchased from Millipore.
DNA transfection. 107 RAW 264.7 (or HL60) cells were transfected as previously described (42) with $10 \mu \mathrm{g}$ of mTRAF6 (Invivogen), flag-tagged mTRAF2, flag-tagged mTRAF3, or the respective control plasmids. To determine transfection efficiency, all cells were also transfected with pMaxGFP plasmid (Amaxa). Cells were then plated in 24-well plates in RPMI (supplemented with $10 \%$ FCS, L-glutamine, and gentamycin). $20 \mathrm{~h}$ after transfection, cells were exposed to $\mathrm{LXA}_{4}$ or vehicle control and incubated for a further $24 \mathrm{~h}$ before stimulation with LPS, CD40L, TNF, or STAg. Cells and supernatants were harvested $24 \mathrm{~h}$ after stimulation and analyzed for GFP expression (FACS), IL-12 expression (ELISA), and expression of the transfected proteins (Western blot).

Statistical analysis. The statistical significance of differences in mean values between experimental versus control or vehicle-treated samples was evaluated by means of Student's $t$ test. Differences were considered to be significant at $\mathrm{P}<0.05$.

Online supplemental material. Fig. S1 shows that TRAF2 and TRAF6 are molecular targets for LX-mediated, SOCS2-dependent inhibition of TLR and TNFR family signaling. Fig. S2 shows that late in vivo treatment with PR11 during LCMV infection does not affect the priming of LCMVspecific CD8+ T cells. Fig. S3 shows the cytokine expression in the presence of proteasome inhibition during infection with $T$. gondii. Figs. S4-9 shows the in situ immunofluorescence analysis of SOCS2 induction, TRAF2 and TRAF6 expression, and pro-inflammatory gene expression after proteasome inhibition during T. gondii infection of WT and SOCS2-deficient mice. Fig. S10 shows that proteasome inhibition blocks ATL-induced SOCS2 antiinflammatory actions in vivo. Fig. S11 shows that ASA-induced SOCS2 targets TRAF2 and blocks p50 and p65 translocation to the nucleus after LPS stimulation. The online version of this article is available at http://www .jem.org/cgi/content/full/jem.20072416/DC1.

We thank Drs. W. Alexander, M. Ripberger, and L. Zhenggang for providing us with SOCS2-deficient mice, IL-10-deficient mice, and TRAF2- and 3-containing plasmids, respectively. We also thank Ms. S. Wojciechowski for technical assistance with the LCMV infection model.

This work was supported by a grant from CCHMC (Trustee Grant/CFF-RDP Center) to J. Aliberti, along with grants from National Institutes of Health to J. Aliberti (Al075038), C.L. Karp (HL079312), and C.N. Serhan (GM38765 and P50-DED16191).

The authors declare no conflicting financial interests.

Submitted: 13 November 2007

Accepted: 19 March 2008

\section{REFERENCES}

1. Aliberti, J., C. Serhan, and A. Sher. 2002. Parasite-induced lipoxin $A_{4}$ is an endogenous regulator of IL-12 production and immunopathology in Toxoplasma gondii infection. J. Exp. Med. 196:1253-1262.

2. Bafica, A., C.A. Scanga, C. Serhan, F. Machado, S. White, A. Sher, and J. Aliberti. 2005. Host control of Mycobacterium tuberculosis is regulated by 5-lipoxygenase-dependent lipoxin production. J. Clin. Invest. 115:1601-1606.

3. Karp, C.L., L.M. Flick, K.W. Park, S. Softic, T.M. Greer, R. Keledjian, R. Yang, J. Uddin, W.B. Guggino, S.F. Atabani, et al. 2004. Defective lipoxin-mediated anti-inflammatory activity in the cystic fibrosis airway. Nat. Immunol. 5:388-392.

4. Kieran, N.E., P. Maderna, and C. Godson. 2004. Lipoxins: potential antiinflammatory, proresolution, and antifibrotic mediators in renal disease. Kidney Int. 65:1145-1154.

5. Samuelsson, B. 1991. Arachidonic acid metabolism: role in inflammation. Z. Rheumatol. 50:3-6.

6. Van Dyke, T.E., and C.N. Serhan. 2003. Resolution of inflammation: a new paradigm for the pathogenesis of periodontal diseases. J. Dent. Res. 82:82-90.

7. Serhan, C.N. 2007. Resolution phase of inflammation: novel endogenous anti-inflammatory and proresolving lipid mediators and pathways. Anпu. Rev. Immunol. 25:101-137. 
8. Celik, G.E., F.O. Erkekol, Z. Miotasiotarliotagil, and M. Melli. 2007. Lipoxin A(4) levels in asthma: relation with disease severity and aspirin sensitivity. Clin. Exp. Allergy. 37:1494-1501.

9. Machado, F.S., J.E. Johndrow, L. Esper, A. Dias, A. Bafica, C.N. Serhan, and J. Aliberti. 2006. Anti-inflammatory actions of lipoxin A4 and aspirin-triggered lipoxin are SOCS-2 dependent. Nat. Med. 12:330-334.

10. Yoshimura, A., T. Naka, and M. Kubo. 2007. SOCS proteins, cytokine signalling and immune regulation. Nat. Rev. Immunol. 7:454-465.

11. Bradley, J.R., and J.S. Pober. 2001. Tumor necrosis factor receptor-associated factors (TRAFs). Oncogene. 20:6482-6491.

12. Chung, J.Y., Y.C. Park, H. Ye, and H. Wu. 2002. All TRAFs are not created equal: common and distinct molecular mechanisms of TRAFmediated signal transduction. J. Cell Sci. 115:679-688.

13. Xu, L.G., L.Y. Li, and H.B. Shu. 2004. TRAF7 potentiates MEKK3induced AP1 and CHOP activation and induces apoptosis. J. Biol. Chem. 279:17278-17282

14. Cao, Z., W.J. Henzel, and X. Gao. 1996. IRAK: a kinase associated with the interleukin-1 receptor. Science. 271:1128-1131.

15. Leo, E., K. Welsh, S. Matsuzawa, J.M. Zapata, S. Kitada, R.S. Mitchell, K.R. Ely, and J.C. Reed. 1999. Differential requirements for tumor necrosis factor receptor-associated factor family proteins in CD40-mediated induction of NF-kappaB and Jun N-terminal kinase activation. J. Biol. Chem. 274:22414-22422.

16. Nakano, H., H. Oshima, W. Chung, L. Williams-Abbott, C.F. Ware, H. Yagita, and K. Okumura. 1996. TRAF5, an activator of NF-kap$\mathrm{paB}$ and putative signal transducer for the lymphotoxin-beta receptor. J. Biol. Chem. 271:14661-14664.

17. Nishitoh, H., M. Saitoh, Y. Mochida, K. Takeda, H. Nakano, M. Rothe, K. Miyazono, and H. Ichijo. 1998. ASK1 is essential for JNK/ SAPK activation by TRAF2. Mol. Cell. 2:389-395.

18. Rothe, M., V. Sarma, V.M. Dixit, and D.V. Goeddel. 1995. TRAF2mediated activation of NF-kappa B by TNF receptor 2 and CD40. Science. 269:1424-1427.

19. Mason, N.J., J. Fiore, T. Kobayashi, K.S. Masek, Y. Choi, and C.A. Hunter. 2004. TRAF6-dependent mitogen-activated protein kinase activation differentially regulates the production of interleukin-12 by macrophages in response to Toxoplasma gondii. Infect. Immun. 72:5662-5667.

20. Rico-Bautista, E., A. Flores-Morales, and L. Fernandez-Perez. 2006 Suppressor of cytokine signaling (SOCS) 2, a protein with multiple functions. Cytokine Growth Factor Rev. 17:431-439.

21. Kile, B.T., B.A. Schulman, W.S. Alexander, N.A. Nicola, H.M. Martin, and D.J. Hilton. 2002. The SOCS box: a tale of destruction and degradation. Trends Biochem. Sci. 27:235-241.

22. Orr, S.J., N.M. Morgan, R.J. Buick, C.R. Boyd, J. Elliott, J.F. Burrows, C.A. Jefferies, P.R. Crocker, and J.A. Johnston. 2007. SOCS3 targets Siglec 7 for proteasomal degradation and blocks Siglec 7-mediated responses. J. Biol. Chem. 282:3418-3422.

23. Reis e Sousa, C., S. Hieny, T. Scharton-Kersten, D. Jankovic, H. Charest, R.N. Germain, and A. Sher. 1997. In vivo microbial stimulation induces rapid CD40 ligand-independent production of interleukin 12 by dendritic cells and their redistribution to T cell areas. J. Exp. Med. 186:1819-1829

24. Bao, J., K. Sato, M. Li, Y. Gao, R. Abid, W. Aird, M. Simons, and M.J. Post. 2001. PR-39 and PR-11 peptides inhibit ischemia-reperfusion injury by blocking proteasome-mediated I kappa B alpha degradation. Am. J. Physiol. Heart Circ. Physiol. 281:H2612-H2618.

25. Reis e Sousa, C., G. Yap, O. Schulz, N. Rogers, M. Schito, J. Aliberti, S. Hieny, and A. Sher. 1999. Paralysis of dendritic cell IL-12 production by microbial products prevents infection-induced immunopathology. Immunity. 11:637-647.

26. Aliberti, J., S. Hieny, C. Reis e Sousa, C.N. Serhan, and A. Sher. 2002. Lipoxin-mediated inhibition of IL-12 production by DCs: a mechanism for regulation of microbial immunity. Nat. Immunol. 3:76-82.

27. Serhan, C.N., S.D. Brain, C.D. Buckley, D.W. Gilroy, C. Haslett, L.A O’Neill, M. Perretti, A.G. Rossi, and J.L. Wallace. 2007. Resolution of inflammation: state of the art, definitions and terms. FASEB J. 21:325-332.

28. Banchereau, J., F. Briere, C. Caux, J. Davoust, S. Lebecque, Y.J. Liu, B. Pulendran, and K. Palucka. 2000. Immunobiology of dendritic cells. Annu. Rev. Immunol. 18:767-811.

29. Aliberti, J., C. Reis e Sousa, M. Schito, S. Hieny, T. Wells, G.B Huffnagle, and A. Sher. 2000. CCR 5 provides a signal for microbial induced production of IL-12 by CD8 alphat dendritic cells. Nat. Immunol. 1:83-87.

30. Aliberti, J., J.G. Valenzuela, V.B. Carruthers, S. Hieny, J. Andersen, H. Charest, C. Reis e Sousa, A. Fairlamb, J.M. Ribeiro, and A. Sher. 2003 Molecular mimicry of a CCR 5 binding-domain in the microbial activation of dendritic cells. Nat. Immunol. 4:485-490.

31. Floto, R.A., P.A. MacAry, J.M. Boname, T.S. Mien, B. Kampmann, J.R. Hair, O.S. Huey, E.N. Houben, J. Pieters, C. Day, et al. 2006. Dendritic cell stimulation by mycobacterial Hsp70 is mediated through CCR 5. Science. 314:454-458

32. Aliberti, J. 2005. Host persistence: exploitation of anti-inflammatory pathways by Toxoplasma gondii. Nat. Rev. Immunol. 5:162-170.

33. Machado, F.S., and J. Aliberti. 2006. Impact of lipoxin-mediated regulation on immune response to infectious disease. Immunol. Res. 35: 209-218.

34. Santiago, H.C., C.G. Feng, A. Bafica, E. Roffe, R.M. Arantes, A. Cheever, G. Taylor, L.Q. Vieira, J. Aliberti, R.T. Gazzinelli, and A. Sher. 2005. Mice deficient in LRG-47 display enhanced susceptibility to Trypanosoma cruzi infection associated with defective hemopoiesis and intracellular control of parasite growth. J. Immunol. 175:8165-8172.

35. Mackowiak, P.A. 2000. Brief history of antipyretic therapy. Clin. Infect. Dis. 31:S154-S156.

36. Vane, J.R. 1971. Inhibition of prostaglandin synthesis as a mechanism of action for aspirin-like drugs. Nat. New Biol. 231:232-235.

37. Abramson, S., H. Korchak, R. Ludewig, H. Edelson, K. Haines, R.I. Levin, R. Herman, L. Rider, S. Kimmel, and G. Weissmann. 1985. Modes of action of aspirin-like drugs. Proc. Natl. Acad. Sci. USA. 82: 7227-7231

38. Claria, J., and C.N. Serhan. 1995. Aspirin triggers previously undescribed bioactive eicosanoids by human endothelial cell-leukocyte interactions. Proc. Natl. Acad. Sci. USA. 92:9475-9479.

39. Kopp, E., and S. Ghosh. 1994. Inhibition of NF-kappa B by sodium salicylate and aspirin. Science. 265:956-959.

40. Paul-Clark, M.J., T. Van Cao, N. Moradi-Bidhendi, D. Cooper, and D.W. Gilroy. 2004. 15-epi-lipoxin $\mathrm{A}_{4}$-mediated induction of nitric oxide explains how aspirin inhibits acute inflammation. J. Exp. Med. 200:69-78.

41. Grunvald, E., M. Chiaramonte, S. Hieny, M. Wysocka, G. Trinchieri, S.N. Vogel, R.T. Gazzinelli, and A. Sher. 1996. Biochemical characterization and protein kinase $\mathrm{C}$ dependency of monokine-inducing activities of Toxoplasma gondii. Infect. Immun. 64:2010-2018.

42. Ma, X., J.M. Chow, G. Gri, G. Carra, F. Gerosa, S.F. Wolf, R. Dzialo, and G. Trinchieri. 1996. The interleukin $12 \mathrm{p} 40$ gene promoter is primed by interferon $\gamma$ in monocytic cells. J. Exp. Med. 183:147-157. 